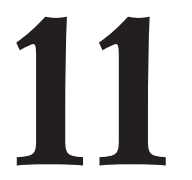

\title{
LOS COSTES NO MONETARIOS DEL ABANDONO EDUCATIVO PREMATURO: UNA ESTIMACIÓN EN TÉRMINOS DE AÑOS DE BUENA SALUD ${ }^{1}$
}

\author{
(THE NON-MONETARY COSTS OF EARLY SCHOOL LEAVING: ESTIMATION \\ IN TERMS OF YEARS OF GOOD HEALTH)
}

Marcos Fernández-Gutiérrez

Universidad de Cantabria

Jorge Calero Martínez

Universidad de Barcelona e IEB

DOI: 10.5944/educxx1.17.2.11490

Cómo referenciar este artículo/How to reference this article:

Fernández Gutiérrez, M. y Calero Martínez, J. (2014). Los costes no monetarios del abandono educativo prematuro: Una estimación en términos de años de buena salud. Educación XX1, 17 (2), 241-263. doi: 10.5944/educxx1.17.2.11490

Fernández Gutiérrez, M. y Calero Martínez, J. (2014). The non-monetary costs of early school leaving: Estimation in terms of years of good health. Educación XX1, 17 (2), 241-263. doi: 10.5944/educxx1.17.2.11490

\section{RESUMEN}

El Abandono Educativo Prematuro (AEP) se ha situado en los últimos años, en España, en cifras cercanas al 30\%, muy alejadas del objetivo del 10\% estipulado por la UE. El AEP da lugar a importantes costes monetarios y no monetarios, de los cuales son de particular importancia los costes en términos de salud, observados tanto en hábitos y comportamientos con repercusiones sobre la misma (inputs) como en los propios resultados en salud (outputs). Las elevadas cifras de AEP han despertado la preocupación de la sociedad y de las políticas educativas; sin embargo, la evaluación de los costes del AEP $\mathrm{y}$, en particular, aquellos de tipo no monetario (tales como los relativos a la salud), no ha sido apenas abordada hasta la fecha. El objetivo de este trabajo es, precisamente, evaluar y cuantificar los costes no monetarios del AEP en términos de la salud futura de la población española. Para ello, se plantea un análisis microeconométrico, que permite aislar el efecto de la educación sobre el estado de salud percibido, a partir de la información de la Encuesta Nacional de Salud; posteriormente, estos resultados se trasladan al conjunto de la población española, con un horizonte temporal de veinte años. Los resultados obtenidos reflejan los notables costes del AEP sobre la salud: las 
personas que han experimentado AEP muestran resultados en salud destacadamente peores, fruto de lo cual tienen una esperanza de vida con buena salud sensiblemente inferior. En consecuencia, resulta necesario incorporar la consideración de estos costes no monetarios del AEP en términos de salud a la toma de decisiones educativas por parte tanto de la ciudadanía como de los poderes públicos, con el fin valorar adecuadamente el problema y los beneficios de las políticas educativas destinadas a afrontarlo.

\section{PALABRAS CLAVE}

Economía de la educación, Política de la educación, Abandono de estudios, Oportunidades educativas, Salud.

\section{ABSTRACT}

Early School Leaving (ESL) has reached rates of around 30\% in Spain, far from the EU target of reducing it to $10 \%$ in 2020. ESL generates significant monetary and non-monetary costs. Among the latter, those in terms of health are of particular importance, observed both in habits and behavior with impact on health (inputs) and in health results (outputs). The high rates of ESL have led to wide concerns among society and the education policy-makers. However, the evaluation of the costs of ESL and, in particular, non-monetary costs (such as those related to health), has not yet been addressed in sufficient depth. This paper aims to evaluate and quantify the non-monetary costs of ESL in terms of the future health outcomes of Spanish population. To this end, a microeconometric analysis is performed, which isolates the effect of education on self-perceived health status, from the information available in the Spanish National Health Survey (Encuesta Nacional de Salud). The results are then extrapolated to the whole Spanish population, with a time horizon of twenty years. The results obtained reflect the remarkable costs of ESL in terms of health: having experienced ESL is strongly related to worse health outcomes and due to this, to a lower life expectancy in good health. Consequently, need arises to incorporate consideration of these non-monetary costs of ESL in terms of health both by the citizens and by the policy makers, thereby permitting an adequate assessment of the problem and the benefits of education policies designed to tackle it.

\section{KEYWORD}

Economics Education, Educational policy, Dropouts, Educational opportunities, Health. 


\section{INTRODUCCIÓN}

El Abandono Educativo Prematuro (AEP en adelante), definido como el porcentaje de personas de entre 18 y 24 años que tienen como estudios más elevados Educación Secundaria Básica y que no se encuentran escolarizados (Council of the European Union, 2009) alcanzó, en España, el 31,9\% en 2008 (EC, 2009; Instituto de Evaluación, 2010). En los últimos años, en gran medida como consecuencia de la reducción de los puestos de trabajo disponibles para la población de esta franja de edad derivada de la crisis económica, el AEP ha disminuido ligeramente en nuestro país, situándose en el 26,5\% en 2011; no obstante, la tasa de AEP en España continúa siendo la segunda mayor de la UE-27 y prácticamente duplica el promedio europeo $(13,5 \%)$ (Instituto Nacional de Evaluación Educativa, 2012). El AEP genera importantes costes, tanto para el individuo que lo experimenta como para el conjunto de la sociedad (EC, 2011; Oreopoulos, 2007; Psacharopoulos, 2007). De los costes individuales, los que se perciben de un modo más inmediato son los de tipo monetario, ligados a la mayor probabilidad de desempleo entre los individuos con AEP y, en caso de estar empleados, a sus menores ingresos esperados. Pero existen, además, una serie de costes no monetarios, a partir de la incidencia del AEP sobre el desarrollo formativo y personal y, de esta forma, sobre un amplio abanico de posteriores comportamientos y hábitos individuales, con efectos sobre la salud, la satisfacción personal, las decisiones en el ámbito personal y familiar (como el nivel educativo que alcanzan los hijos) y el grado de inclusión social. Los costes sociales del AEP, por su parte, se encuentran ligados a la existencia de externalidades negativas derivadas de un menor nivel educativo de la población: menores niveles de productividad, desarrollo tecnológico y riqueza; peor salud de la población; menor equidad y cohesión social; y, asimismo, mayores necesidades de gasto público para afrontar problemas sociales en materia de protección por desempleo, servicios sociales, sanidad, dependencia, seguridad y justicia. Además, las decisiones educativas se enfrentan a problemas de información imperfecta: como ha destacado Oreopoulos (2007), los individuos no conocen con certeza los costes y beneficios futuros asociados al AEP. Ello afecta, en particular, a los costes no monetarios, cuya detección es generalmente más difícil y se demora en mayor medida.

Las externalidades y los problemas de información imperfecta constituyen fallos de mercado que justifican la intervención de las políticas públicas en materia educativa, con el fin de alcanzar niveles educativos socialmente óptimos. Concretamente, la UE, en el marco de la Estrategia de Lisboa, considera la reducción del AEP por debajo del 10\% en 2020 como uno de los objetivos fundamentales para incrementar la competitividad y el dinamismo de la economía, en paralelo a la promoción del desarrollo personal y la cohesión social (Council of the European Union, 2009; EC, 2011). 
En España, las cifras de AEP casi triplican dicho objetivo y se han consolidado como un grave problema educativo, económico y social (EC, 2009), fruto de lo cual su reducción se ha configurado, bajo el impulso del Ministerio de Educación, como una prioridad fundamental de las políticas educativas. Sin embargo, la evaluación de los costes del AEP no ha sido aún suficientemente abordada pese a que, como destaca Psacharopoulos (2007), su conocimiento y estimación (inclusive de aquellos costes de tipo no monetario) resulta fundamental para el desarrollo de las políticas educativas destinadas a su reducción.

El contexto descrito constituye la motivación del objetivo principal de este trabajo: evaluar y cuantificar los costes no monetarios del AEP, en España, en términos de la salud futura de la población, con un horizonte temporal de veinte años. La elección de este ámbito se debe a la relevancia particular de las relaciones entre educación y salud que, como han señalado Groot y Maasen van den Brink (2007) y Grossman (2006), constituyen las dos principales inversiones de los individuos en capital humano. Con ello, pretende contribuirse a incorporar la consideración de los costes no monetarios del AEP a la toma de decisiones educativas y a la implementación de políticas públicas al respecto. El trabajo se estructura de la siguiente manera: tras esta introducción, la siguiente sección define y describe los efectos no monetarios de la educación, focalizando en los aspectos relativos a la salud; a continuación, se detallan los datos y la metodología utilizados en la estimación; tras ello, la siguiente sección recoge los resultados obtenidos y su discusión; finalmente, la última sección incorpora las conclusiones finales derivadas de dichos resultados y sus implicaciones en términos de políticas educativas.

\section{MARCO TEÓRICO}

Los efectos no monetarios de la educación son todas aquellas consecuencias de la adquisición de un determinado nivel educativo no asociadas directamente a la obtención de un beneficio monetario, sino a otros aspectos no monetarios, ligados al desarrollo formativo y personal. De ellos, aquellos que repercuten positivamente sobre el individuo que adquiere el nivel educativo y/o sobre la sociedad que le ofrece dicha posibilidad constituyen los beneficios no monetarios de la educación. Dichos beneficios dejan de obtenerse cuando el individuo no adquiere el nivel educativo en cuestión; ello constituye, en consecuencia, los costes no monetarios del AEP. La literatura se ha centrado en el análisis y evaluación de los efectos monetarios de la educación; sin embargo, los efectos no monetarios, analizados en menor medida fundamentalmente debido a su heterogeneidad y a la mayor complejidad de su detección y valoración, revisten también una enorme im- 
portancia y han de considerarse para desarrollar una evaluación completa de las repercusiones de la educación sobre el bienestar individual y social, como han destacado autores como Albert y Davia (2004), Grossman (2006) y Haveman y Wolfe (1984). De entre los efectos no monetarios de la educación, la literatura ha destacado la relevancia de los relativos a la salud, en un contexto en el que esta depende crecientemente de los estilos de vida, constituidos por un entramado de comportamientos sobre los cuales la educación tiene gran capacidad para influir. Ilustrativamente, de acuerdo con el reciente estudio de Galea et al (2011), en Estados Unidos se producen 245.000 muertes anuales atribuibles a un bajo nivel educativo, el cual, de esta forma, resultaría el factor social con mayores repercusiones en términos de salud.

Desde la economía neoclásica, los efectos no monetarios de la educación sobre la salud se han explicado a partir del incremento en la eficiencia en la producción del output «buena salud» (eficiencia productiva) y en la utilización de los inputs que inciden sobre la salud (eficiencia asignativa) (Grossman, 2006). Como explicación alternativa, la escuela institucionalista se ha centrado en la incidencia de la educación en la configuración del entorno institucional de los individuos (tales como las relaciones y las circunstancias personales, familiares, laborales y sociales) y, de esta manera, en la formación de preferencias individuales, de las cuales se derivan los hábitos de consumo y estilos de vida (Escardíbul y Calero, 2006; Feinstein et al, 2006).

Con independencia de este debate, son numerosas las investigaciones que han reflejado la existencia de efectos no monetarios de la educación sobre la salud, como se recoge en las extensas recopilaciones de la literatura desarrolladas por Feinstein et al (2006) y Grossman (2006). Estos efectos se reflejarían tanto en los hábitos y comportamientos con repercusiones sobre la salud (inputs) como, fruto de ellos, en los propios resultados en salud (outputs). En lo que respecta a los inputs, se ha detectado una incidencia beneficiosa de la educación fundamentalmente en cuatro ámbitos: el uso del sistema sanitario, el consumo de productos perjudiciales, la realización de hábitos beneficiosos y determinadas decisiones residenciales y ocupacionales con repercusiones sobre la salud. En lo relativo al uso del sistema sanitario, las investigaciones realizadas han encontrado cómo el mayor nivel educativo se relaciona con incrementos en el uso de los cuidados preventivos, la atención al tratamiento de problemas crónicos y el recurso a cuidados especializados (Feinstein et al 2006), así como con un menor consumo inapropiado de servicios médicos y productos farmacéuticos (Haveman y Wolfe, 1984). En lo que respecta al consumo de productos perjudiciales para la salud, se ha contrastado cómo el nivel educativo se encuentra inversamente relacionado con la probabilidad de fumar y con la cantidad de cigarrillos fumados diariamente (Bratti y Miranda, 2009). En un análisis 
aplicado al caso de España, Escardíbul y Calero (2006) encontraron que la educación reduce la probabilidad de fumar entre los hombres, aunque no entre las mujeres. Por el contrario, dichos autores no encontraron una relación del nivel educativo con el consumo de alcohol, si bien sí con la probabilidad de realizar un consumo abusivo, en sintonía con los resultados de las investigaciones realizadas en otros países (Grossman y Kaestner, 1997). En cuanto al tercero de los inputs previamente referidos, el nivel educativo se ha demostrado estadísticamente relacionado con la práctica de actividad física, como realizar actividades deportivas con una frecuencia semanal o desplazarse caminando habitualmente, si bien con resultados diferenciados en función del sexo y la edad; también en relación a la alimentación, pese a que existen resultados heterogéneos en función del tipo de nutrientes, se ha contrastado la existencia de una relación positiva entre el nivel educativo y la probabilidad de seguir una dieta equilibrada, en términos de la ingesta de alimentos como las frutas y las verduras y de nutrientes como las vitaminas y la fibra (Feinstein et al, 2006). Finalmente, respecto al cuarto de los grupos de inputs mencionados, la educación incide significativamente, por un lado, en la elección del lugar donde vivir (Vila, 2003), lo que repercute en la salud a través de aspectos como las condiciones generales de habitabilidad, las relaciones con el vecindario y la contaminación del aire; asimismo, en lo que respecta a la ocupación, el nivel educativo se relaciona significativamente con mejores condiciones ambientales y de salud laboral y con una mayor satisfacción con el horario y la distancia y comunicación con el trabajo (García-Mora, 2004), todo ello con potenciales consecuencias sobre la salud.

La educación, debido a su incidencia sobre este conjunto de inputs, tiene también un efecto destacado sobre los resultados o condiciones futuras de salud (outputs). A este respecto, Feinstein et al (2006) han destacado la relación existente entre el nivel educativo y la probabilidad de próxima mortalidad, así como la probabilidad de tener una hospitalización. Ilustrativamente, diferenciando en función de las principales causas de mortalidad, Borrell et al (1999) observaron una asociación inversa, para los hombres, entre el nivel educativo y la probabilidad de fallecer por cáncer de pulmón entre los 45 y los 64 años y por otras enfermedades del sistema respiratorio a partir de dicha edad y, para las mujeres, por enfermedades cerebrovasculares entre los 45 y los 64 años y cardiovasculares a partir de los 65. Más allá de la mortalidad, un mayor nivel educativo tiene repercusiones positivas sobre los resultados en salud relativos a la calidad de vida, tales como la probabilidad de tener limitaciones físicas futuras para realizar actividades habituales (dependencia) y de padecer obesidad (Feinstein et al, 2006). El nivel educativo incide, asimismo, a partir de su efecto sobre los hábitos y comportamientos previamente descritos, no solo sobre la salud propia, sino también sobre la de la familia y el entorno cercano (fundamentalmente, la de los hijos) (Haveman y Wolfe, 1984; Vila, 2003). 
Para el análisis de las repercusiones de la educación sobre los resultados en salud, se encuentra ampliamente extendido el uso del estado de salud percibido, dado que este indicador ha demostrado una aproximación adecuada y consistente del estado de salud real (Feinstein et al, 2006), el cual no es observable directamente (Groot y Maasen van den Brink, 2007). Feinstein et al (2006), Grossman (2006) y Grossman y Kaestner (1997) describen en detalle la literatura que ha encontrado una relación positiva entre el nivel educativo y el estado de salud percibido, lo cual, de acuerdo con Contoyannis y Jones (2004), vendría determinado en gran medida por los hábitos y comportamientos individuales. Ilustrativamente, Oreopoulos (2007) obtuvo, en una investigación focalizada específicamente en el AEP que, en el Reino Unido, un año adicional de permanencia en el sistema educativo reducía en un $3,2 \%$ la probabilidad de declarar tener un mal estado de salud e incrementaba en torno a un 6\% la probabilidad de declarar tener un buen estado de salud. En el ámbito de España, Urbanos (2000), con datos de la Encuesta Nacional de Salud, encontró también una relación positiva entre el nivel educativo y el estado de salud percibido.

La tabla 1 resume, a modo de síntesis, el conjunto de efectos no mo-

netarios del nivel educativo sobre la salud descritos en esta sección. Asimismo, como indicador ilustrativo de todo ello, Groot y Maasen van den Brink (2007), en un análisis aplicado al caso de Holanda, han cuantificado los beneficios sobre la salud de un año adicional de educación, obteniendo que, en términos monetarios, dichos beneficios representarían una cantidad situada en un intervalo de 600-1.380 euros anuales para los hombres y 300690 euros anuales para las mujeres. De acuerdo con esta estimación, los beneficios no monetarios de la educación sobre la salud futura superarían por sí solos a los costes de permanecer dicho año adicional en el sistema educativo y representarían entre el 15 y el 60\% respecto al valor de los beneficios de tipo monetario.

\begin{tabular}{|c|c|c|}
\hline Ámbito & Efecto & Fuente \\
\hline \multirow{4}{*}{$\begin{array}{l}\text { Uso del sistema } \\
\text { sanitario }\end{array}$} & Mayor uso de los cuidados preventivos & \multirow{3}{*}{ Feinstein et al (2006) } \\
\hline & $\begin{array}{l}\text { Mayor atención al tratamiento } \\
\text { de problemas crónicos }\end{array}$ & \\
\hline & $\begin{array}{l}\text { Mayor recurso a cuidados } \\
\text { especializados }\end{array}$ & \\
\hline & $\begin{array}{l}\text { Menor consumo inapropiado } \\
\text { de servicios médicos y productos } \\
\text { farmacéuticos }\end{array}$ & $\begin{array}{l}\text { Haveman y Wolve } \\
(1984)\end{array}$ \\
\hline
\end{tabular}




\begin{tabular}{|c|c|c|}
\hline Ámbito & Efecto & Fuente \\
\hline \multirow{2}{*}{$\begin{array}{l}\text { Consumo } \\
\text { de productos } \\
\text { perjudiciales } \\
\text { para la salud }\end{array}$} & $\begin{array}{l}\text { Menor probabilidad de fumar y menos } \\
\text { cigarrillos fumados diariamente }\end{array}$ & \begin{tabular}{|l|} 
Bratti y Miranda \\
$(2009) ;$ Escardíbul \\
y Calero (2006)
\end{tabular} \\
\hline & Menor consumo abusivo de alcohol & $\begin{array}{l}\text { Escardíbul y Calero } \\
\text { (2006); Grossman } \\
\text { y Kaestner (1997) }\end{array}$ \\
\hline \multirow{2}{*}{$\begin{array}{l}\text { Realización } \\
\text { de hábitos } \\
\text { beneficiosos } \\
\text { para la salud }\end{array}$} & $\begin{array}{l}\text { Mayor práctica habitual de actividad } \\
\text { física }\end{array}$ & \multirow[b]{2}{*}{ Feinstein et al (2006) } \\
\hline & $\begin{array}{l}\text { Mayor probabilidad de seguir una } \\
\text { dieta adecuada (frutas, verduras, } \\
\text { vitaminas, fibra, etc) }\end{array}$ & \\
\hline \multirow{3}{*}{\begin{tabular}{|l} 
Decisiones \\
residenciales \\
y ocupacionales \\
con repercusio- \\
nes sobre la salud
\end{tabular}} & $\begin{array}{l}\text { Elección del lugar de residencia } \\
\text { y las condiciones de habitabilidad, } \\
\text { relaciones con el vecindario y } \\
\text { contaminación del aire }\end{array}$ & Vila (2003) \\
\hline & $\begin{array}{l}\text { Mejores condiciones ambientales } \\
\text { y de salud en el trabajo }\end{array}$ & \multirow[b]{2}{*}{ García-Mora (2004) } \\
\hline & $\begin{array}{l}\text { Mayor satisfacción con el horario } \\
\text { y la distancia y comunicación con el } \\
\text { trabajo }\end{array}$ & \\
\hline \multirow{6}{*}{$\begin{array}{l}\text { Resultados } \\
\text { objetivos en salud }\end{array}$} & $\begin{array}{l}\text { Menor probabilidad de próxima } \\
\text { mortalidad }\end{array}$ & \multirow[t]{2}{*}{ Feinstein et al (2006) } \\
\hline & Menor probabilidad de hospitalización & \\
\hline & $\begin{array}{l}\text { Menor probabilidad de mortalidad } \\
\text { por determinadas enfermedades }\end{array}$ & Borrell et al (1999) \\
\hline & $\begin{array}{l}\text { Menor probabilidad de padecer } \\
\text { limitaciones físicas para realizar } \\
\text { actividades habituales }\end{array}$ & \multirow[t]{2}{*}{ Feinstein et al (2006) } \\
\hline & $\begin{array}{l}\text { Menor probabilidad de padecer } \\
\text { obesidad }\end{array}$ & \\
\hline & $\begin{array}{l}\text { Mejor salud de la familia y el entorno } \\
\text { cercano }\end{array}$ & $\begin{array}{l}\text { Haveman y Wolve } \\
\text { (1984); Vila (2003) }\end{array}$ \\
\hline $\begin{array}{l}\text { Resultados } \\
\text { subjetivos en } \\
\text { salud }\end{array}$ & Mejor estado de salud percibido & $\begin{array}{l}\text { Grossman y Kaestner } \\
\text { (1997); Urbanos } \\
\text { (2000); Contoyannis } \\
\text { y Jones (2004); } \\
\text { Feinstein et al (2006); } \\
\text { Grossman (2006); } \\
\text { Oreopoulos (2007) }\end{array}$ \\
\hline
\end{tabular}

Tabla 1. Resumen de los principales efectos no monetarios de un mayor nivel educativo sobre la salud 


\section{METODOLOGía}

Para abordar el objetivo del estudio, con un horizonte de veinte años vista, se plantea que la actual cohorte poblacional en fase de tomar sus decisiones educativas (bajo el supuesto simplificador de que estas se toman entre los 16 y 22 años de edad), así como aquellas que lo harán en los próximos veinte años, dejan de experimentar AEP y toman una decisión educativa alternativa. De esta manera, se simulan los efectos de una hipotética política educativa que implicará la eliminación completa del AEP en el periodo 2008-2027, frente a la situación prevista bajo el mantenimiento de los parámetros actuales. Dado que no es posible conocer el comportamiento futuro de los individuos, se parte del supuesto de que reproducirán, dadas sus decisiones educativas, los comportamientos observados actualmente para las mismas franjas de edad. El cálculo se lleva a cabo en dos fases: en primer lugar se estiman, a nivel individual, los efectos de las decisiones educativas sobre la salud; a continuación, dichas estimaciones se extrapolan al conjunto de la población objetivo, a partir de un hipotético cambio en sus decisiones educativas que implique la desaparición total del AEP.

En la primera fase, la estimación se lleva a cabo de manera diferenciada para las siguientes franjas de edad: 16-22 años, en la cual el grueso de los individuos toman sus decisiones educativas y la mayor parte de aquellos que cursan estudios adicionales a la educación obligatoria continúa en el sistema educativo, como se desprende de INE (2007); 23-35 años, franja que finaliza cuando han transcurrido veinte años desde el comienzo de la toma de las decisiones educativas analizadas; 36-42 años, que abarca hasta el momento en que han transcurrido veinte años desde el final del primer intervalo; 43-65 años, franja que finaliza con la edad legal de jubilación; y, finalmente, de 66 años en adelante. La fuente utilizada es la Encuesta Nacional de Salud (en adelante, ENS) (MSPS, 2006), a partir de la cual se obtiene una muestra útil de 26.205 individuos de los que se dispone de información completa para las estimaciones planteadas. El amplio tamaño muestral de esta encuesta y el diseño de la muestra, cuyas características se detallan en el documento metodológico específico del que dispone la ENS (MSPS, 2006) permiten ofrecer una elevada representatividad; incluso, como se describe posteriormente, resulta factible plantear estimaciones separadas para las submuestras correspondientes a distintas franjas de edad. Además, la ENS incorpora factores de elevación poblacional, cuya utilización permite obtener resultados representativos no ya de la propia muestra, sino del conjunto de la población española.

Respecto al principal elemento de interés del análisis (las decisiones educativas), la ENS ofrece información sobre el nivel educativo completado, desagregado en nueve categorías y, además, permite identificar si el 
individuo, en el momento considerado, es estudiante o no. Mediante todo ello, se construye la variable que recoge sus decisiones educativas, detallada en la tabla 2. Como se observa, de acuerdo con la definición de AEP, quedan englobados en dicha situación aquellos cuyo máximo nivel de estudios sea inferior a la primera etapa de Educación Secundaria y aquellos que, habiendo completado dicho nivel, no se encuentren estudiando. Los restantes individuos se diferencian en dos grupos: aquellos que han cursado o cursan un nivel educativo adicional (opción que se denomina Decisión 1), bien Formación Profesional de Grado Medio o bien la segunda etapa de Educación Secundaria; y aquellos que han continuado más allá de dicho nivel adicional (opción denominada Decisión 2), estudiando Educación Superior (Formación Profesional de Grado Superior o Estudios Universitarios).

La tabla 3 muestra la distribución de la población española en función de dichas decisiones educativas, por franja de edad. Como se observa, el AEP es creciente con la edad y resulta particularmente elevado en las franjas de 43 a 65 años y de más de 65 años. Sin embargo, las diferencias entre las franjas de mayor y menor edad en las decisiones educativas y la incidencia del AEP han de valorarse teniendo en cuenta que las decisiones educativas se derivan en gran medida del contexto sociocultural en el que se toman y este ha experimentado cambios muy significativos a lo largo del tiempo. Esta puntualización, no obstante, no afecta al núcleo de los resultados obtenidos en este artículo dado que, debido al horizonte de veinte años planteado en el análisis, los cambios extrapolados a la población objetivo en la segunda fase de la estimación se derivan de los resultados correspondientes a las tres franjas de menor edad (hasta 42 años), donde las decisiones educativas sí son en gran medida comparables.

\begin{tabular}{|l|l|l|}
\hline \multicolumn{1}{|c|}{ Nivel educativo alcanzado } & \multicolumn{1}{c|}{ ¿Es estudiante? } & \multirow{2}{*}{ Decisión educativa } \\
\hline Analfabeto & - & \multirow{2}{*}{ AEP } \\
\hline Sin Estudios Completos & - & \\
\hline Estudios Primarios & - & \\
\hline \multirow{2}{*}{ Ed. Secundaria, primera etapa } & No & \multirow{2}{*}{ DECISIÓN 1 } \\
\cline { 2 - 3 } & Sí & \\
\hline \multirow{2}{*}{ FP Grado Medio } & No & DECISIÓN 2 \\
\cline { 2 - 3 } & Sí & \multicolumn{2}{|l}{} \\
\hline
\end{tabular}




\begin{tabular}{|l|l|l|}
\hline \multicolumn{1}{|c|}{ Nivel educativo alcanzado } & \multicolumn{1}{|c|}{ ¿Es estudiante? } & \multirow{2}{*}{ Decisión educativa } \\
\hline \multirow{2}{*}{ Ed. Secundaria, segunda etapa } & No & \multirow{2}{*}{ DECISIÓN 1 } \\
\cline { 2 - 2 } & Sí & \\
\cline { 1 - 2 } FP Grado Superior & - & \multirow{2}{*}{ DECISIÓN 2 } \\
\hline E. Universitaria, primer ciclo & - & \\
\hline E. Universitaria, Segundo ciclo & - & \\
\hline
\end{tabular}

Tabla 2. Diferenciación de los individuos en función de sus decisiones educativas

\begin{tabular}{|l|c|c|c|c|c|c|}
\hline & $\begin{array}{c}\text { De 16 } \\
\text { a 22 }\end{array}$ & $\begin{array}{c}\text { De 23 } \\
\text { a 35 }\end{array}$ & $\begin{array}{c}\text { De 36 } \\
\text { a 42 }\end{array}$ & $\begin{array}{c}\text { De 43 } \\
\text { a 65 }\end{array}$ & $\begin{array}{c}\text { Más } \\
\text { de 65 }\end{array}$ & TOTAL \\
\hline AEP & 29,58 & 34,90 & 42,76 & 60,62 & 88,06 & 53,72 \\
\hline Decisión 1 & 32,87 & 26,27 & 25,85 & 17,85 & 4,85 & 20,12 \\
\hline Decisión 2 & 37,54 & 38,83 & 31,39 & 21,53 & 7,09 & 26,16 \\
\hline
\end{tabular}

Tabla 3. Distribución porcentual de la muestra utilizada por niveles educativos y franja de edad

Fuente: elaboración propia a partir de MSPS (2006)

En las estimaciones, se incluyen una serie de variables de control disponibles en la ENS, que permiten separar del efecto correspondiente a las decisiones educativas el ligado a los siguientes factores: el sexo, mediante la variable «Mujer»; la nacionalidad, a partir de las variables «ExtranjUE»y "ExtranjNoUE», que permiten diferenciar, respectivamente, a los extranjeros de otro país de la UE y de fuera de la misma; el carácter urbano o rural del lugar de residencia, mediante la variable "Urbano», que hace referencia a los municipios de más de 50.000 habitantes; los ingresos del hogar, a partir de seis intervalos y las variables «Ing601a900», "Ing901a1200», "Ing1201a1800», "Ing1801a3600» y "IngMas3600», frente a los ingresos inferiores a 601 euros anuales (categoría de referencia); y el número de miembros del hogar (variables «UnaPersona», "TresPersonas», "CuatroPers» y "MasCuatroPers», frente a dos personas como categoría de referencia). Asimismo, las estimaciones incorporan los factores de ponderación poblacional incluidos en la ENS, ofreciendo así resultados representativos a nivel de la población española.

La variable dependiente analizada es la probabilidad de tener un buen estado de salud declarado, así entendido cuando el encuestado percibe su 
estado de salud como «bueno» o «muy bueno»; mientras, la mala salud se infiere a partir del estado de salud percibido como «regular», «malo» $\mathrm{O}$ «muy malo", como propone el Ministerio de Sanidad (MSPS, 2009). De acuerdo con la ENS, el 66,6\% de la población española cuenta con un buen estado de salud declarado, cifra que decrece sensiblemente con la edad: $89,1 \%$ para la franja de $16-22$ años; $81,3 \%$ para la de $23-35$ años; $75,1 \%$ para la de $36-42$ años; 60,8\% para la de 43-65 años y 39\% para la de más de 65 años.

Con todo ello se llevan a cabo, para cada franja de edad, las siguientes estimaciones probit, bajo el supuesto de que la variable dependiente sigue, en cada una de ellas, una distribución normal estándar $\square$ :

$$
\operatorname{Pr}\left(y_{i}\right)=\Phi\left(d_{i}, x_{i}\right)
$$

Donde:

$y_{\mathrm{i}}=$ probabilidad de tener un buen estado de salud declarado.

$d_{i}=$ decisión educativa del individuo i. Se estima el efecto marginal asociado a las variables "Decisión1» y "Decisión2», que indica la variación porcentual esperada en la probabilidad de tener un buen estado de salud declarado como consecuencia de cada una de dichas decisiones educativas, en relación a la situación de AEP, para cada franja de edad y una vez corregidos los efectos de las variables de control.

$x_{\mathrm{i}}=$ vector de variables de control.

Estas estimaciones permiten contrastar, para cada franja de edad, si las decisiones educativas consideradas incrementan, frente al AEP, la probabilidad de tener un buen estado de salud declarado y, en su caso, cuantificar los efectos existentes. Esta cuantificación parte del supuesto de caeteris paribus; esto es, se estima el efecto de las decisiones educativas sobre el estado de salud declarado bajo el supuesto de que todas las demás variables permanecen constantes. A partir de la cuantificación de dichos efectos, para completar esta primera fase de la estimación, se calcula el efecto esperado de cada una de las decisiones educativas alternativas al AEP en términos de esperanza de vida con buena salud, definida como el número medio de años que espera vivir con buena salud una persona a una edad determinada (MSPS, 2009). El cálculo se realiza a partir de los efectos marginales previamente estimados de las decisiones educativas sobre la probabilidad de que un individuo tenga un buen estado de salud declarado en cada edad a lo largo de su vida. Trasladando dichos efectos marginales estimados a las cifras actuales de esperanza de vida con buena salud de la población española (MSPS, 2009) y a partir de la descrita definición de esperanza de vida con buena salud, se obtiene el incremento asociado a la Decisión 1 y a la 
Decisión 2, respecto a la situación de AEP, en el número de años que, para cada edad a lo largo de su vida, un individuo esperará vivir declarando tener buena salud.

Tras ello, en la segunda fase del análisis empírico, se estiman los costes totales del AEP en términos de salud para el conjunto de la población objetivo. Para ello, en primer lugar, se calcula la distribución de la población de entre 18 y 24 años en función de sus decisiones educativas, en la situación actual y en una hipotética situación simulada. En esta última, bajo la premisa de desaparición absoluta del AEP, podrían ocurrir dos alternativas extremas. De acuerdo con el escenario más optimista, cabría considerar que los individuos en situación de AEP no cuentan con ningún elemento diferenciador que incida sobre sus decisiones educativas; en ese caso, una vez desapareciera la situación de AEP, estos individuos se repartirían entre las Decisiones 1 y 2 en una proporción idéntica a la de aquellos que no experimentan AEP. De acuerdo con el escenario más pesimista, cabría considerar un determinismo absoluto, en el cual los individuos en situación de AEP contarían con una serie de características particulares por las cuales no podrían alcanzar la decisión educativa más avanzada (Decisión 2); en este escenario, todos los individuos que dejaran la situación de AEP pasarían a tomar la Decisión 1, pero ninguno la Decisión 2. En el presente análisis, se considera un escenario intermedio entre ambos, que tiene en cuenta una serie de características particulares de los individuos en situación de AEP (tales como el lugar de residencia y el menor nivel educativo medio de sus progenitores) que condicionarán también sus decisiones educativas una vez abandonen dicha situación pero, simultáneamente, evita el determinismo absoluto por el cual ninguno de ellos alcanzaría la decisión 2. De esta forma y a partir de un análisis de las variables que inciden en las decisiones educativas, el escenario intermedio seleccionado considera que los individuos con AEP, una vez abandonen dicha situación, pasarían a tomar la Decisión 2 con una probabilidad igual a dos tercios de la de aquellos que no experimentan AEP y la Decisión 1 en otro caso. Bajo este supuesto, se estima la magnitud de la población de entre 18 y 24 años que, fruto de los cambios simulados, pasaría de una situación de AEP a la Decisión 1 o la Decisión 2. Esta segunda fase de la estimación se lleva a cabo a partir de la muestra de individuos de la Encuesta de Presupuestos Familiares (EPF) de 2007 (INE, 2007), cuya utilización viene motivada por su amplitud y representatividad (la muestra incluye 61.676 individuos), así como por ofrecer la información apropiada, en formato de microdatos, para analizar las decisiones educativas planteadas y realizar de una manera precisa el cálculo descrito.

Para el cálculo de los efectos con un horizonte de veinte años vista, las decisiones educativas y los cambios simulados para la población de entre 18 y 24 años en 2007 se extrapolan al conjunto de cohortes poblacionales cuyos 
individuos tomarán sus decisiones educativas entre 2008 y 2027 (esto es, los nacidos entre 1985 y 2011). Con ello, se estima el número total de individuos que, bajo la hipotética desaparición absoluta del AEP en este intervalo de veinte años, pasarían de una situación de AEP a la Decisión 1 o a la Decisión 2. Seguidamente, a partir de estas cifras y de los valores previamente obtenidos en las estimaciones a nivel individual, se calcula el número de personas que pasarían a tener un buen estado de salud declarado y, con ello, una aproximación a la valoración monetaria del coste total del AEP en términos de salud.

\section{RESULTADOS Y DISCUSIÓN}

La tabla 4 muestra los resultados relativos a la primera fase del análisis empírico: los efectos marginales estimados de las decisiones educativas sobre la probabilidad de tener un buen estado de salud declarado, como indicador de los resultados en salud. Como se observa, tanto la Decisión 1 como la Decisión 2 incrementan, de manera intensa y ampliamente significativa, la probabilidad de tener un buen estado de salud declarado en todas las franjas de edad (como única excepción, la significatividad del efecto de la Decisión 1 en el segmento de 36 a 42 años no alcanza el 90\%); en todas ellas, el efecto de la Decisión 2 es más intenso que el de la Decisión 1. La intensidad de estos efectos se incrementa con la edad, reflejo del carácter acumulativo de gran parte de los elementos que inciden sobre los resultados en salud. De esta forma, se estima que la Decisión 1 aumenta, respecto a la situación de AEP, la probabilidad de tener un buen estado de salud declarado en: un 4,4\% para la franja de 16-22 años; un 5,2\% para la de 23-35; un $9,6 \%$ para la de $43-65$ y un $13,2 \%$ para la de mayores de 65 . Mientras, respecto al AEP, la Decisión 2 incrementa dicha probabilidad en: un 7\% para la franja de 16-22 años; un 9\% para la de 23-35; un 9,1\% para la de 36-42; un $15,9 \%$ para la de $43-65$ y un $16,2 \%$ para la de mayores de 65 . Estos resultados son consistentes con los obtenidos por Oreopoulos (2007), si bien los derivados del presente estudio dependen de la decisión educativa y de la franja de edad. Finalmente, respecto a las variables de control, destaca cómo el sexo femenino reduce la probabilidad de tener un buen estado de salud declarado, mientras que un mayor nivel de ingresos la incrementa notablemente, especialmente en las franjas de mayor edad.

Las decisiones educativas repercuten no solo sobre el estado de salud declarado, sino sobre un conjunto amplio de hábitos y comportamientos de riesgo para la salud, como se ha detallado con anterioridad, a través de lo cual acaban incidiendo en los resultados en salud. Con carácter ilustrativo, las estimaciones descritas para el estado de salud declarado se han reproducido manteniendo como variables independientes las decisiones educativas y el resto de variables de control y tomando como variables dependientes, 
a partir de la información recogida en la ENS, la probabilidad de incidencia de cada uno de los cinco principales comportamientos de riesgo, de acuerdo con la OMS, en términos de causa de pérdida de salud en los países desarrollados (OMS, 2002; Feinstein et al., 2006): el tabaco, el alcohol, el sobrepeso, la dieta inadecuada y la falta de actividad física. Los resultados obtenidos, que se omiten por razones de espacio, reflejan cómo las decisiones educativas alternativas al AEP, en mayor medida la Decisión 2, reducen intensa y significativamente la probabilidad de fumar (excepto en las franjas de mayor edad) y de no realizar alguna actividad física habitualmente; asimismo, la Decisión 2, si bien no la Decisión 1, reduce significativamente la probabilidad de no seguir una dieta adecuada. Respecto al sobrepeso, las decisiones educativas alternativas al AEP también reducen, en las franjas de edad intermedia, la probabilidad de declarar tener un peso «normal» recogida en la ENS. Finalmente y como excepción, las decisiones educativas no muestran un efecto significativo sobre el consumo de alcohol, lo cual podría reflejar, de acuerdo con Escardíbul y Calero (2006), la influencia del entorno y el contexto social, ligados al nivel educativo, sobre el comportamiento de los individuos, que explicaría que no siempre un mayor nivel educativo conduzca a mejores hábitos de salud en todos los aspectos. Con independencia de esta puntualización, los efectos de las decisiones educativas alternativas al AEP sobre los principales hábitos y comportamientos de riesgo para la salud se encuentran en consonancia con los estimados en términos del estado de salud declarado y, de hecho, constituyen un relevante elemento explicativo de los mismos.

\begin{tabular}{|c|c|c|c|c|c|c|}
\hline & & \multicolumn{5}{|c|}{ Buen Estado de Salud Declarado } \\
\hline & & \multicolumn{5}{|c|}{ Edad } \\
\hline & & $16-22$ & $23-35$ & $36-42$ & $43-65$ & Más 65 \\
\hline \multirow[t]{3}{*}{ Educación } & Decisión 1 & $\begin{array}{l}0,044 * * \\
(0,006,0,083)\end{array}$ & $\begin{array}{l}0,052 * * * \\
(0,019,0,085)\end{array}$ & $\begin{array}{l}0,033 \\
(-0,119,0,078)\end{array}$ & $\begin{array}{l}0,096 * * * \\
(0,058,0,134)\end{array}$ & $\begin{array}{l}0,132 * * * \\
(0,047,0,217)\end{array}$ \\
\hline & Decisión 2 & $\begin{array}{l}0,070 * * * \\
(0,029,0,111)\end{array}$ & $\begin{array}{l}0,090 * * * \\
(0,057,0,124)\end{array}$ & $\begin{array}{l}0,091 * * * \\
(0,047,0,135)\end{array}$ & $\begin{array}{l}0,159 * * * \\
(0,121,0,197)\end{array}$ & $\begin{array}{l}0,162^{* * *} \\
(0,087,0,237)\end{array}$ \\
\hline & Mujer & $\begin{array}{l}-0,087 * * * \\
(-0,127,-0,047)\end{array}$ & $\begin{array}{l}-0,061 * * * \\
(-0,090,-0,032)\end{array}$ & $\begin{array}{l}-0,077 * * * \\
(-0,114,-0,040)\end{array}$ & $\begin{array}{l}-0,104 * * * \\
(-0,132,-0,076)\end{array}$ & $\begin{array}{l}-0,135 * * * \\
(-0,170,-0,101)\end{array}$ \\
\hline \multirow[t]{5}{*}{$\begin{array}{l}\text { Variables } \\
\text { de control }\end{array}$} & ExtranjUE & $\begin{array}{l}-0,084 \\
(-0,225,0,058)\end{array}$ & $\begin{array}{l}0,022 \\
(-0,047,0,090)\end{array}$ & $\begin{array}{l}0,070 \\
(-0,037,0,177)\end{array}$ & $\begin{array}{l}-0,058 \\
(-0,174,0,058)\end{array}$ & $\begin{array}{l}0,030 \\
(-0,167,0,227)\end{array}$ \\
\hline & ExtranjNoUE & $\begin{array}{l}-0,067 \\
(-0,149,0,015)\end{array}$ & $\begin{array}{l}-0,052^{*} \\
(-0,104,0,000)\end{array}$ & $\begin{array}{l}-0,067^{*} \\
(-0,145,0,010)\end{array}$ & $\begin{array}{l}0,038 \\
(-0,038,0,115)\end{array}$ & $\begin{array}{l}0,152 \\
(-0,117,0,420)\end{array}$ \\
\hline & Urbano & $\begin{array}{l}-0,028 \\
(-0,065,0,010)\end{array}$ & $\begin{array}{l}-0,015 \\
(-0,044,0,015)\end{array}$ & $\begin{array}{l}-0,014 \\
(-0,051,0,023)\end{array}$ & $\begin{array}{l}0,006 \\
(-0,023,0,035)\end{array}$ & $\begin{array}{l}0,029^{*} \\
(-0,005,0,063)\end{array}$ \\
\hline & Ing601a900 & $\begin{array}{l}0,018 \\
(-0,055,0,091)\end{array}$ & $\begin{array}{l}0,014 \\
(-0,062,0,091)\end{array}$ & $\begin{array}{l}0,045 \\
(-0,044,0,134)\end{array}$ & $\begin{array}{l}0,089 * * * \\
(0,033,0,145)\end{array}$ & $\begin{array}{l}0,015 \\
(-0,029,0,060)\end{array}$ \\
\hline & Ing901a1200 & $\begin{array}{l}0,027 \\
(-0,041,0,096)\end{array}$ & $\begin{array}{l}0,046 \\
(-0,020,0,113)\end{array}$ & $\begin{array}{l}0,064 \\
(-0,016,0,144)\end{array}$ & $\begin{array}{l}0,152 * * * \\
(0,101,0,203)\end{array}$ & $\begin{array}{l}0,086 * * * \\
(0,034,0,139)\end{array}$ \\
\hline
\end{tabular}




\begin{tabular}{|c|c|c|c|c|c|}
\hline & \multicolumn{5}{|c|}{ Buen Estado de Salud Declarado } \\
\hline & \multicolumn{5}{|c|}{ Edad } \\
\hline & $16-22$ & $23-35$ & $36-42$ & $43-65$ & Más 65 \\
\hline Ing1201a1800 & $\begin{array}{l}0,056^{*} \\
(-0,008,0,120)\end{array}$ & $\begin{array}{l}0,053 \\
(-0,014,0,119)\end{array}$ & $\begin{array}{l}0,045 \\
(-0,039,0,128)\end{array}$ & $\begin{array}{l}0,195 * * * \\
(0,145,0,246)\end{array}$ & $\begin{array}{l}0,096 * * * \\
(0,034,0,159)\end{array}$ \\
\hline Ing1801a3600 & $\begin{array}{l}0,061^{*} \\
(-0,002,0,124)\end{array}$ & $\begin{array}{l}0,085 * * * \\
(0,021,0,149)\end{array}$ & $\begin{array}{l}0,129 * * * \\
(0,053,0,205)\end{array}$ & $\begin{array}{l}0,250 * * * \\
(0,200,0,300)\end{array}$ & $\begin{array}{l}0,156^{* * *} \\
(0,071,0,241)\end{array}$ \\
\hline IngMas 3600 & $\begin{array}{l}0,069 * * * \\
(0,024,0,115)\end{array}$ & $\begin{array}{l}0,075^{* *} \\
(0,003,0,147)\end{array}$ & $\begin{array}{l}0,129 * * * \\
(0,051,0,207)\end{array}$ & $\begin{array}{l}0,217 * * * \\
(0,152,0,283)\end{array}$ & $\begin{array}{l}0,293^{* * *} \\
(0,110,0,476)\end{array}$ \\
\hline UnaPersona & $\begin{array}{l}0,025 \\
(-0,055,0,106)\end{array}$ & $\begin{array}{l}0,015 \\
(-0,037,0,068)\end{array}$ & $\begin{array}{l}0,013 \\
(-0,063,0,089)\end{array}$ & $\begin{array}{l}0,053 * * \\
(0,010,0,095)\end{array}$ & $\begin{array}{l}0,050 * * * \\
(0,013,0,088)\end{array}$ \\
\hline TresPersonas & $\begin{array}{l}0,026 \\
(-0,027,0,079)\end{array}$ & $\begin{array}{l}-0,034^{*} \\
(-0,071,0,004)\end{array}$ & $\begin{array}{l}0,005 \\
(-0,054,0,064)\end{array}$ & $\begin{array}{l}0,002 \\
(-0,033,0,037)\end{array}$ & $\begin{array}{l}-0,009 \\
(-0,061,0,043)\end{array}$ \\
\hline CuatroPers & $\begin{array}{l}0,042 \\
(-0,015,0,098)\end{array}$ & $\begin{array}{l}-0,001 \\
(-)\end{array}$ & $\begin{array}{l}0,027 \\
(-0,029,0,083)\end{array}$ & $\begin{array}{l}0,030 \\
(-0,007,0,068)\end{array}$ & $\begin{array}{l}0,010 \\
(-0,079,0,099)\end{array}$ \\
\hline $\begin{array}{l}\text { MasCuatro- } \\
\text { Pers }\end{array}$ & $\begin{array}{l}0,042 \\
(-0,014,0,098)\end{array}$ & $\begin{array}{l}-0,047^{*} \\
(-0,104,0,009)\end{array}$ & $\begin{array}{l}-0,064^{*} \\
(-0,140,0,011)\end{array}$ & $\begin{array}{l}-0,048^{*} \\
(-0,102,0,006)\end{array}$ & $\begin{array}{l}-0,096 * * \\
(-0,190,0,003)\end{array}$ \\
\hline $\mathbf{N}$ & 1.414 & 5.024 & 3.822 & 9.308 & 6.637 \\
\hline Wald chi2 & 61,22 & 94,28 & 98,84 & 381,88 & 171,76 \\
\hline Prob > chi2 & 0,000 & 0,000 & 0,000 & 0,000 & 0,000 \\
\hline
\end{tabular}

Tabla 4. Efectos marginales estimados sobre la probabilidad de tener un buen estado de salud declarado

Significatividad: $*>90 \%, * *>95 \%, * * *>99 \%$

Entre paréntesis, intervalos de confianza con un nivel de significatividad del 95\%

Fuente: elaboración propia a partir de MSPS (2006).

A partir de los efectos marginales de las decisiones educativas sobre la probabilidad de tener un buen estado de salud declarado, se calcula el incremento en la esperanza de vida con buena salud asociado a cada decisión educativa, en relación al AEP, de la forma descrita en el apartado metodológico. Concretamente, los resultados obtenidos previamente para cada franja de edad implican que, con las actuales cifras de esperanza de vida con buena salud (MSPS, 2009) y tasas de mortalidad, para un individuo medio de entre 16 y 22 años, la Decisión 1 supone, respecto al AEP, un incremento estimado de 3,9 años en su esperanza de vida con buena salud, mientras que la Decisión 2 supone un incremento de 6,6 años. A los 35 años, el incremento estimado de esperanza de vida con buena salud es de 3,1 años con la Decisión 1 (que supone, al menos, cursar 2 años adicionales de educación respecto al AEP) y de 5,3 años con la Decisión 2 (que supone entre 4 y 6 años adicionales respecto al AEP). Estos resultados pueden compararse con el obtenido por Lleras-Muney (2005), quien, en un análisis aplicado al caso de Estados Unidos ha estimado que, en 1960, un año adicional de 
educación incrementaba la esperanza de vida a los 35 años de edad (en la versión más habitual del indicador, sin corregir por la calidad de vida) en 1,7 años. Las estimaciones del presente trabajo son consistentes con las de Lleras-Muney (2005); aunque estas últimas resultan algo más elevadas, ello puede responder a que ambos estudios difieren en cuanto a indicador utilizado, país y periodo.

Los datos obtenidos en la primera fase de la estimación se extrapolan, en una segunda fase, al conjunto de la población objetivo. El análisis de la situación real previa a los cambios simulados en las decisiones educativas indica que, de los 3.768.226 españoles de entre 18 y 24 años en 2007, 1.203.476 individuos (el 31,94\%) experimentaban AEP, mientras que 922.305 (el 24,48\%) habían optado por la Decisión 1 y 1.642 .445 (el 43,59\%) por la Decisión 2 (INE, 2007). A partir de esas cifras y del escenario intermedio utilizado en la simulación, 513.797 de las personas en situación de AEP pasarían a tomar la Decisión 2 (dos tercios de la frecuencia con que toman dicha decisión los restantes individuos), mientras las 689.679 restantes optarían por la Decisión 1. De esta forma, tras los cambios simulados, el $42,78 \%$ de la población de entre 18 y 24 años habría optado por la Decisión 1 y el 57,22\% por la Decisión 2, mientras que el AEP habría desaparecido completamente. Dichos porcentajes se trasladan, a continuación, al total de cohortes afectadas por una eliminación del AEP en el periodo 2008-2027, a partir de las cifras reales de población y proyecciones poblacionales del INE (2010a, 2011). Como excepción, tanto en la situación real prevista como tras los cambios simulados, las dos cohortes más jóvenes (con 16 y 17 años, respectivamente, al finalizar 2027), únicamente habrán podido tomar la Decisión 1. La tabla 5 resume, para cada una de las franjas de edad diferenciadas, la distribución de la población objetivo en función de sus decisiones educativas en la situación real prevista, las cifras afectadas por los cambios simulados ligados a la eliminación completa del AEP y, fruto de ello, la hipotética distribución de la población objetivo en función de sus decisiones educativas tras la simulación.

\begin{tabular}{|l|l|r|r|r|r|r|}
\cline { 3 - 7 } \multicolumn{2}{c|}{} & \multicolumn{2}{c|}{$\begin{array}{c}\text { Situación real } \\
\text { prevista }\end{array}$} & $\begin{array}{c}\text { Cambio si- } \\
\text { mulado }\end{array}$ & \multicolumn{2}{c|}{$\begin{array}{c}\text { Situación final } \\
\text { simulada }\end{array}$} \\
\cline { 3 - 8 } & Número & $\%$ & Número & Número & $\%$ \\
\hline \multirow{3}{*}{$\begin{array}{l}\text { Franja de } 16 \\
\text { a 22 años }\end{array}$} & AEP & 1.106 .194 & 31,94 & -1.106 .194 & 0 & 0,00 \\
\cline { 2 - 8 } & Decisión 1 & 1.282 .407 & 37,02 & 769.900 & 2.052 .307 & 59,25 \\
\cline { 2 - 8 } & Decisión 2 & 1.075 .022 & 31,04 & 336.294 & 1.411 .316 & 40,75 \\
\cline { 2 - 7 } & TOTAL & 3.463 .623 & 100,00 & 0 & 3.463 .623 & 100,00 \\
\hline
\end{tabular}




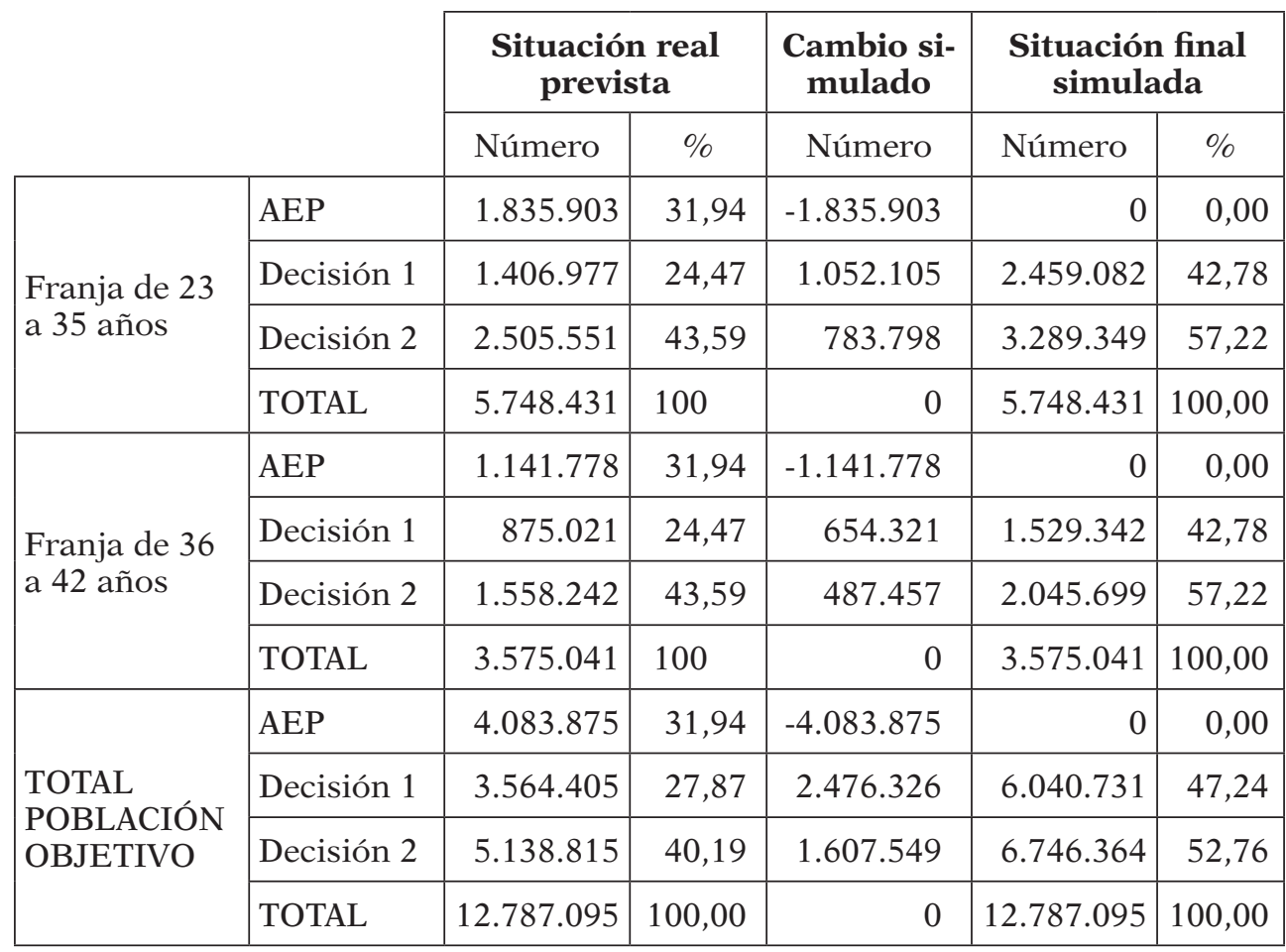

Tabla 5. Cambios simulados en las decisiones educativas de la población objetivo

Fuente: Elaboración propia a partir de INE (2007, 2010a, 2011)

Estas cifras, junto a los resultados obtenidos en la primera parte del análisis empírico implican que, como consecuencia de la desaparición del AEP durante un periodo de veinte años, al finalizar 2027, el número de personas con un buen estado de salud declarado se habría incrementado en 226.977 personas. Esta cifra representa, en consecuencia, el coste del AEP en términos de pérdida de salud de la población española con un horizonte de veinte años vista. En caso de aumentar el horizonte temporal considerado, dicha cifra se incrementaría sensiblemente, dado que los efectos se han observado crecientes con la edad.

Para cuantificar en términos monetarios el valor de este coste sobre la salud, se utilizan las estimaciones de Pinto-Prades, Loomes y Brey (2009) acerca del valor marginal de un año de vida ajustado por su calidad (QALY), a partir de la disposición a pagar por distintos tratamientos. Estos autores, en una estimación en la que tratan de evitar los sesgos en la valoración motivados por el orden de las respuestas, obtienen un rango amplio en la cuantificación (en función de las características de cada tratamiento), que 
va desde los 16.400 euros hasta los 123.700 euros. En el presente artículo, con el fin de maximizar el realismo de la estimación, se opta por un escenario intermedio que se deriva del promedio de los valores estimados por estos autores, a partir de lo cual se obtiene un valor monetario aproximativo de un año de vida con buena salud, de $50.000 €$. Este valor conduce a cuantificar el coste no monetario del AEP en términos de resultados en salud en el año 2027 en 11.348,84 millones de euros a precios actuales, lo cual representa en torno al 1,04\% del PIB español de 2008 (INE, 2010b).

\section{CONCLUSIONES Y REFLEXIONES FINALES}

Este trabajo contribuye a poner de manifiesto la importancia de los costes no monetarios del AEP en un ámbito fundamental para la calidad de vida, como la salud, a partir de su estimación y cuantificación para el caso de España. Las estimaciones planteadas han permitido obtener evidencia de cómo, frente a la situación de AEP, la adquisición de niveles educativos más elevados repercute enormemente en los resultados en salud. Concretamente, se observa un notable incremento en la probabilidad de tener un buen estado de salud declarado asociado a la Decisión 1 (completar un nivel educativo más allá de la educación obligatoria: FP de Grado Medio o Educación Secundaria Superior) y a la Decisión 2 (completar otro nivel educativo adicional: FP de Grado Superior o Educación Universitaria) que, además, se muestra creciente con la edad. Este resultado, consistente con otras investigaciones realizadas en el ámbito de España, como las de Urbanos (2000), se derivaría de los efectos de la educación sobre un amplio conjunto de hábitos y comportamientos con repercusiones sobre la salud que han sido detectados por la literatura científica, como se ha descrito al inicio del presente trabajo.

Los resultados obtenidos implican que, en el momento de tomar las decisiones educativas, la Decisión 1 reporta, frente al AEP, 3,9 años más de esperanza de vida con buena salud, mientras que el incremento asociado a la Decisión 2 es de 6,6 años. Se trata de una pérdida enorme para las personas que experimentan AEP, a añadir a las de tipo monetario. Este coste no monetario, sin embargo, es frecuentemente infravalorado o ignorado por los individuos en la toma de decisiones educativas, debido a problemas de información imperfecta, asociados a la difícil detección y la dilación en el tiempo de los efectos sobre la salud. Para el conjunto de la sociedad española, el coste estimado del AEP se traduce en que 226.977 personas dejarían de tener un buen estado de salud declarado en 2027, cuya valoración monetaria resultaría superior al 1\% del PIB anual. La eliminación del AEP generaría, de esta forma, al posibilitar una mejora del nivel de salud de la población, no solo un gran beneficio personal para los individuos que abandonen la situación de AEP, sino también un conjunto de externalidades 
positivas o beneficios sociales, no considerados por los individuos al tomar sus decisiones educativas, tales como menores necesidades de gasto público para atender problemas sanitarios y situaciones de dependencia y una mayor productividad laboral, especialmente en las franjas de mayor edad.

La existencia de estos dos fallos de mercado (externalidades positivas y problemas de información imperfecta) justifica la intervención de las políticas educativas para alcanzar niveles educativos del conjunto de la población socialmente óptimos. En este sentido, los resultados obtenidos constituyen un argumento adicional en favor de consolidar y profundizar las políticas públicas destinadas a la reducción del AEP en España, uno de los más altos de la UE-27. A este respecto, aunque la mayor mejora de la esperanza de vida con buena salud se encuentra asociada a la Decisión 2, el mayor rendimiento marginal de un año adicional de educación se observa ligado a la adquisición de los primeros niveles educativos más allá de los obligatorios (Decisión 1). Ello ilustra la importancia de alcanzar mejoras marginales de los niveles educativos frente a la situación de AEP, como argumento contrario a la «sobrecualificación» que, inevitablemente, generarían supuestamente todas las políticas destinadas a incrementar el nivel educativo de la población.

Finalmente, de cara al diseño de políticas públicas para reducir el AEP, quedan por realizar dos puntualizaciones adicionales. En primer lugar, aunque el cálculo de los costes totales del AEP en términos de salud se ha basado en un supuesto hipotético de desaparición completa del mismo, la gran magnitud de dichos costes hace que también su eliminación parcial (por ejemplo, la reducción en dos tercios de los niveles actuales en España, de acuerdo con el objetivo de la UE) generaría enormes beneficios sobre la salud futura de la población. En segundo lugar, es relevante reseñar que la reducción progresiva del AEP es una condición necesaria, pero no suficiente, para la desaparición de sus costes no monetarios. Esta no resultará automática, sino que dependerá en gran medida de que las personas que superen la situación de AEP cuenten con el resto de factores condicionantes similares a los de los individuos con niveles educativos más elevados, con el fin de poder reproducir también sus hábitos y resultados. Por ello, en particular, se requiere acompañar las políticas educativas para la reducción del AEP con la promoción y creación de empleo de calidad y el fomento de la equidad y la cohesión social, objetivos que, a su vez, la reducción del AEP favorecería alcanzar.

\section{NOTAS}

1 Los autores desean expresar su agradecimiento al Ministerio de Educación por el apoyo financiero recibido para realizar esta investigación, a través del Instituto de Formación del Profesorado, Investigación e Innovación Educativa y del Programa de Formación del Profesorado Universitario (FPU), en el cual participó uno de los autores en el inicio de la investigación. 


\section{REFERENCIAS BIBLIOGRÁFICAS}

Albert, C. y Davia, M. A. (2004). Salud, salarios y educación. Hacienda Pública Española, 169, 11-34.

Borrell, C.; Regidor, E.; Arias, L. C.; Navarro, P.; Puigpinós, R.; Domínguez, V. y Plasència, A. (1999). Inequalities in mortality according to educational level in two large Southern European cities. International Journal of Epidemiology, 28, 58-63.

Bratti, M. y Miranda, A. (2009). Non-Pecuniary Returns to Higher Education: The Effect on Smoking Intensity in the UK. Health Economics, 19(8), 906-920.

Contoyannis, P. y Jones, A. M. (2004). Socioeconomic Status, Health and Lifestyle. Health Economics, 23(5), 965-995.

Council of the European Union (2009). Council conclusions of 12 May 2009 on a strategic framework for European cooperation in education and training ('ET 2020') (2009/C 119/02).

EC (European Commission) (2009). Progress towards the Lisbon Objectives in Education and Training. Indicators and Benchmarks. Brussels: Comission Staff Working Document.

EC (European Commission) (2011). Tackling early school leaving: A key contribution to the Europe 2020 Agenda. Brussels: Communication from the Commission to the European Parliament, the Council, the European Economic and Social Committee and the Committee of the Regions.

Escardíbul, J. O. y Calero, J. (2006). Educación, estilo de vida y salud: un estudio aplicado al caso español. Revista de Educación, 339, 541-562.

Feinstein, L.; Sabates, R.; Anderson, T. M.; Sorhaindo, A. y Hammond, C.
(2006). What are the effects of education on health? En: R. Desjardins y T. Schuller (eds.) Measuring the effect of education on health and civic engagement. (pp. 171-310). Paris: Organization for Economic Cooperation and Development.

Galea, S.; Tracy, M.; Hoggatt, K. J.; DiMaggio, C. y Karpati, A. (2011). Estimated Deaths Attributable to Social Factors in the United States. American Journal of Public Health, 101(8), 14491455.

García-Mora, M. B. (2004). Efectos de la educación sobre los determinantes de la satisfacción laboral en España. Un análisis de los beneficios monetarios y no monetarios en el mercado de trabajo mediante modelos logit ordenados. Tesis doctoral, Universitat de Valencia.

Groot, W. y Maasen Van Den Brink, H. (2007). The health effects of education. Economics of Education Review, 26(2), 186-200.

Grossman, M. (2006). Education and nonmarket outcomes. En: E. Hanushek (ed.) Handbook of the Economics of Education. (pp. 577-633). Amsterdam: Elsevier.

Grossman, M. y Kaestner, R. (1997). Effects of Education on Health. En: J.R. Behrman y N. Stacey (eds.) The social benefits of education. (pp. 69-124). Ann Arbor: Michigan University Press.

Haveman, R. H. y Wolfe, B. L. (1984). Schooling and economic well-being: The role of nonmarket effects. The Journal of Human Resources, 19(3), 377-407.

Instituto de Evaluación (2010). Sistema Estatal de Indicadores de la Educación. Madrid: Ministerio de Educación. Re- 
cuperado de: http://www.mecd.gob. es/dctm/ievaluacion/indicadores-educativos/seie-2010-completo-imprenta. pdf?documentId=0901e72b8062e4f6

Instituto Nacional de Estadística (2007). Encuesta de Presupuestos Familiares. Madrid: INE.

Instituto Nacional de Estadística (2010a). Proyecciones de Población a Largo Plazo. Madrid: INE.

Instituto Nacional de Estadística (2010b). Contabilidad Nacional de España. Madrid: INE.

Instituto Nacional de Estadística (2011). Estimaciones de la Población Actual. Madrid: INE.

Instituto Nacional de Evaluación Educativa (2012). Sistema Estatal de Indicadores de la Educación. Madrid: Ministerio de Educación, Cultura y Deporte. Recuperado de: http://www. mecd.gob.es/dctm/inee/indicadoreseducativos/seie2012.pdf?documentId $=0901 \mathrm{e} 72 \mathrm{~b} 81477552$.

Lleras-Muney, A. (2005). The Relationship between Education and Adult Mortality in the United States. Review of Economic Studies, 72(1), 189-221.

Ministerio de Sanidad, Política Social e Igualdad (2006). Encuesta Nacional de Salud. Madrid: MSPS.

Ministerio de Sanidad, Política Social e Igualdad (2009). Indicadores de salud
2009. Evolución de los indicadores del estado de salud e España y su magnitud en el contexto de la Unión Europea. Madrid: MSPS. Recuperado de: http:// www.msps.es/estadEstudios/estadisticas/inforRecopilaciones/indicadoresSalud.htm

Organización Mundial de la Salud (2002). The World Health Report 2002. Reducing. Risks, Promoting Healthy Life. Ginebra: OMS.

Oreopoulos, P. (2007). Do dropouts drop out too soon? Wealth, health and happiness from compulsory schooling. Journal of Public Economics, 91(1112), 2213-2229.

Pinto-Prades, J. L.; Loomes, G. y Brey, R. (2009). Trying to estimate a monetary value for the QALY. Journal of Health Economics, 28, 553-562.

Psacharopoulos, G. (2007). The Costs of School Failure a Feasibility Study. Analytical Report for the European Commission prepared by the European Expert Network on Economics of Education (EENEE).

Urbanos, R. (2000). Desigualdades sociales en salud y efectividad potencial de las políticas públicas: un estudio aplicado con datos españoles. Hacienda Pública Española, 154, 217-238.

Vila, L. E. (2003). Los beneficios no monetarios de la educación. Revista de Educación, 331, 309-324. 


\section{PERFIL ACADÉMICO Y PROFESIONAL DE LOS AUTORES}

Marcos Fernández Gutiérrez, Profesor Ayudante Doctor de Economía Aplicada de la Universidad de Cantabria. Previamente, ha sido Investigador predoctoral en la Universidad de Cantabria, a través del Programa de Formación de Profesorado Universitario (FPU). Ha sido también Investigador visitante en la Universitá degli Studi di Milano (Italia) e Investigador postdoctoral en la University of Exeter (Reino Unido). Sus campos de especialización son la Economía de los Servicios Públicos, la Economía del Consumo y la Economía de la Educación.

Jorge Calero, Catedrático de Economía Aplicada de la Universidad de Barcelona. Es coordinador del GIPE, Grupo Interdisciplinar de Análisis de Política Educativa, grupo consolidado de investigación. Ha sido presidente del Consejo Superior de Evaluación de Cataluña (2010-2011). Ha sido miembro (en el grupo de expertos) del Consejo de Trabajo, Económico y Social de Cataluña (2005-2010). Ha sido Presidente de la Asociación de Economía de la Educación (2002-2004). Sus campos de especialización son la Economía de la Educación y la Economía del Estado del Bienestar, con especial referencia a la economía del gasto público y a la evaluación de las políticas educativas.

Dirección de los autores: Marcos Fernández Gutiérrez

Departamento de Economía

Facultad de Ciencias Económicas

y Empresariales

Avenida de Los Castros, s/n

39005 Santander

E-mail: marcos.fernandez@unican.es

Jorge Calero Martínez

Departamento de Economía Pública

Economía Política y Economía Española

Facultad de Economía y Empresa

Avenida Diagonal, 690

08034 Barcelona

E-mail: jorge.calero@ub.edu

Fecha Recepción del Artículo: 03. Agosto. 2011

Fecha Modificación Artículo: 12. Julio. 2012

Fecha Aceptación del Artículo: 16. Agosto. 2012

Fecha de Revisión para publicación: 09. Enero. 2014 